\title{
Syncytin is involved in breast cancer-endothelial cell fusions
}

Bjerregaard, Bolette; Holck, S.; Christensen, I.J.; Larsson, Lars-Inge

Published in:

Cellular and Molecular Life Sciences

DOI:

$10.1007 / \mathrm{s} 00018-006-6201-9$

Publication date:

2006

Document version

Publisher's PDF, also known as Version of record

Citation for published version (APA):

Bjerregaard, B., Holck, S., Christensen, I. J., \& Larsson, L-I. (2006). Syncytin is involved in breast cancerendothelial cell fusions. Cellular and Molecular Life Sciences, 63(16), 1906-1911.

https://doi.org/10.1007/s00018-006-6201-9 


\section{Syncytin is involved in cancer-endothelial cell fusions}

Bolette Bjerregaard $^{1}$, Susanne Holck ${ }^{2}$ and Ib Jarle Christensen ${ }^{3}$ and Lars-Inge Larsson ${ }^{1}$

${ }^{1}$ Anatomy and Cell Biology, IBHV, KVL, Frederiksberg and Departments of

${ }^{2}$ Pathology and ${ }^{3}$ Surgery, Hvidovre University Hospital, Copenhagen, Denmark

Running title: Syncytin in cancer-endothelial cell fusions

Presented in part at The Symposium on Cell Fusion, Epigenetics and Cancer,

Soderkoping, Sweden, October 13-14, 2005

Proofs and correspondence to:

Lars-Inge Larsson, MD, D.Sc.

Anatomy and Cell Biology, IBHV

KVL

Gronnegaardsvej 7

DK-1870 Frederiksberg C

Copenhagen

Denmark

Phone: +45 35282851

Fax: $\quad+4535282547$

E-mail: lail@kvl.dk 


\begin{abstract}
Cancer cells are able to spontaneously fuse with normal host cells, including endothelial cells, and such fusions may strongly modulate the biological behaviour of tumors. However, the underlying mechanisms are unknown. We now show that human breast cancer cell lines and over a third of human breast cancers express syncytin, an endogenous retroviral envelope protein, previously implicated in fusions between placental trophoblast cells. Additionally, endothelial cells and cancer cells are shown to express ASCT-2, a receptor for syncytin. Syncytin antisense treatment decreases syncytin expression and inhibits fusions between breast cancer cells and endothelial cells. Moreover, a syncytin inhibitory peptide also inhibits fusions between cancer cells and endothelial cells. These results are the first to show that syncytin is expressed by human cancer cells and is involved in cancer-endothelial cell fusions.
\end{abstract}

Key-words: Syncytin, breast cancer, endothelial cells, fusions, ASCT-2 


\section{Introduction}

A number of cell fusions occur during normal development. These include the fusion between the sperm and the egg cell and continue with fusions between placental trophoblasts to form syncytiotrophoblasts, between myoblasts to form muscle fibers and between macrophages to form osteoclasts [1]. Recently, it was shown that the human endogenous retroviral envelope protein syncytin (env-W) is involved in fusions between placental trophoblasts $[2,3]$. Such fusions result in the formation of syncytiotrophoblasts and have been shown to involve the expression of a syncytin receptor, the neutral amino acid transporter ASCT-2, also referred to as the D-type retroviral receptor [2]. Although many endogenous retroviral sequences have degenerated during evolution, the syncytin sequence appears to have been conserved due to its ability to contribute to reproductive functions. Several studies have shown that cancer cells may spontaneously fuse with normal host cells in vitro (4, 5, 6-9) and in vivo [5, 10-13]. Such fusions result in cell hybrids containing an admixture of the parental genomes $[5,11]$. In hybrids formed from fusions induced to occur between cancer cells and normal cells tumorogenicity is usually suppressed [14-18], due to activation of tumor suppressor genes contributed by the normal fusion partner [reviewed in 18]. However, in some cases, as for instance the formation of hybridomas producing monoclonal antibodies, tumorogenicity is not suppressed [19, for overviews see 4,20 , 21]. Moreover, tumor suppression has been shown to be absent in hybrids formed from cells having a dominant negative mutation in p53 or expressing specific oncogenes [22]. The mechanisms by which cancer cells can spontaneously fuse with host cells are currently not understood, but are important since such fusions modify the biological behaviour of the ensuing hybrids. Moreover, the possible occurrence of spontaneous fusions betweeen cancer cells themselves [8] may contribute to aneuploidy and altered biological behaviour. 
Previously, we reported that human breast cancer cells are able to spontaneously fuse with endothelial cells to form hybrid cells expressing protein and chromosomal markers characteristic of both parent cell lines [5]. Initially, heterokaryons appeared but, subsequently, mitotic figures and synkaryons, containing an admixture of the parental genomes in a single nucleus were observed [5]. In addition, studies in nude mice revealed that intravenous injection of metastasizing breast cancer (MDA-MB-231) cells resulted in the formation of hybrid cells containing both human and mouse chromosomes and showing endothelial cell characteristics [5]. Recent data suggest that similar fusions may also occur in man [reviewed in 4, 20 and 21]. We now demonstrate that the fusogenic protein syncytin is expressed in breast cancers and in breast cancer cell lines and also show that endothelial cells, as well as cancer cells, express the syncytin receptor ASCT-2. Moreover, antisense-mediated downregulation of syncytin expression, as well as a syncytin inhibitory peptide, inhibits fusions between breast cancer cells and endothelial cells. This demonstrates that the requirements for cancerendothelial cell fusions are present in many cases of human breast cancer and provides direct proof that syncytin is involved in such fusions. 


\section{Materials and methods}

\section{Cell culture}

MCF-7, MDA-MB-231, human umbilical vein endothelial cells (HUVECs) and calf pulmonary artery endothelial (CPAE) cells were cultured as described [5, 23]. MCF-7 cells were transfected with a previously characterized [25] phosphorthioate-protected syncytin antisense oligonucleotide (env-W 758-777) or with a scrambled control oligonucleotide [25] using oligofectamine (Invitrogen, Carlsbad, CA). After 48 h, MCF7 cells were either extracted for protein and RNA or were trypsinized, allowed to recover for $1 \mathrm{~h}$ and then co-cultured for $24 \mathrm{~h}$ with HUVECs or CPAE cells in chamber slides (Nalge Nunc Int., Naperville, IL). Additionally, MCF-7 cells were cocultured with HUVECs for $24 \mathrm{~h}$ in the presence of $5 \mu \mathrm{g} / \mathrm{ml}$ of a synthetic syncytin peptide (RossPetersen, Hørsholm, Denmark), previously shown to inhibit syncytin-mediated fusions [24]. Control cells were incubated with equivalent concentrations of a control peptide (C-peptide(3-33), Sigma, St. Louis, MO) as well as without any peptide. Cells were fixed in $3.7 \%$ paraformaldehyde in phosphate-buffered saline and permeabilized in $0.1 \%$ triton $\mathrm{X}-100$ or were left unpermeabilized.

\section{Immunocytochemistry and cell fusion assays}

Tumors from 165 female patients with ductal breast cancers without axillary lymph node involvement were fixed in $10 \%$ buffered formalin and embedded in paraffin. Deparaffinized sections underwent antigen retrieval by microwaving and were stained with rabbit antisera to the extracellular domain of syncytin (pab-10109, Orbigen, San Diego, CA) or to ASCT-2 (Chemicon, Temecula, CA). The site of antigen-antibody reaction was revealed by biotin-labeled antirabbit IgG followed by peroxidase-labeled streptavidin (Dako, Glostrup, Denmark) and development in diaminobenzidine. Nuclei were counterstained with haematoxylin. Controls included use of antisera preabsorbed 
with the immunizing peptide (syncytin peptide was custom-synthesized by RossPetersen and the ASCT-2 peptide was purchased from Chemicon). Monoclonal antiCD31 antibodies (Dako) were used in combination with rabbit anti-ASCT-2 antibodies, using detection with species-specific antibodies labeled with Alexa-594 or Alexa-488 (Molecular Probes, Eugene, OR), respectively. Live MCF-7 cells and MCF-7 cells, fixed without permeabilization, were stained with the syncytin antiserum followed by Alexa-488-labeled antirabbit IgG (Molecular Probes). For quantitating cell fusions, specimens were stained for double immunofluorescence using monoclonal antibodies recognizing vimentin (3B4, IgG2a) and cytokeratin (pancytokeratin; IgG1) (Dako) followed by subclass-specific second antibodies labeled with Alexa-488 (anti-IgG2a) or Alexa-594 (anti-IgG1) (Molecular Probes) [5]. Nuclei were stained with bisbenzimide (Hoechst 33258, Sigma). Controls, including use of type-matched monoclonal antibodies, as well as conventional staining controls were negative. Fused cells (cytokeratin and vimentin double-positive cells) were counted in blind-coded specimens and results were expressed as a percentage of fused cells versus unfused cells. In each experiment between 8 and 13 coded samples of each kind were counted and each experiment was repeated at least twice. The Mann-Whitney U-test was used for statistical comparisons.

\section{SDS-PAGE and immunoblotting}

Extraction, SDS-PAGE and electroblotting were performed as described [26]. Antibodies to syncytin (Orbigen) and to HER-2 (Pharmingen, BD Biosciences, Heidelberg, Germany) were used in combination with chemiluminescent detection and the intensities of the bands quantitated using NIH Image software [26]. Syncytin protein levels were expressed relative to HER-2 protein levels.

Reverse Transcriptase-Polymerase Chain Reaction (RT-PCR): Total RNA was prepared using the TRIZOL reagent. Reverse transcription employed the Superscript II 
kit (Life Technologies) following the manufacturer's recommendation for random hexamer RT and PCR except that 2.5 units of AmpliTaq polymerase were used. Forty cycles of PCR (annealing temperature $57^{\circ} \mathrm{C}, 1.5 \mathrm{mM} \mathrm{MgCl}_{2}$ ) were used. For detection of syncytin transcripts primer A (CCGCTGAAAGAGGGGGAA) was used as upstream and primer B (TAGAGGTTGTGCAGCTGAGA) was used as downstream primer [27], producing an amplicon of 400 base pairs while bovine ASCT-2 was amplified using primer C (TGCTGACACCTGTGAGAAGG) as upstream and primer D (CACAGCCACAGCCACTAAAA) as downstream primer, producing an amplicon of 491 base pairs. Human ASCT-2 was amplified using primer E (GACCGTACGGAGTCGAGAAG) as upstream and primer F (GGGACGCAGCAGAACATTAT) as downstream primer, producing an amplicon of 445 base pairs. PCR products were size fractionated by agarose gel electrophoresis. Controls for lack of genomic contamination included omission of RT from the reaction as well as use of intron-spanning primers detecting Hras (primer G: CCAGCTGATCCAGAACCATT and primer H: AGCCAGGTCACACTTGTTCC), producing an amplicon of 301 base pairs from cDNA and of 702 base pairs from genomic DNA, respectively. PCR products were sequenced by DNA Technology (Aarhus, Denmark).

\section{Real-time RT-PCR}

PCR was performed on a LightCycler (Roche) using SYBR green I Fast start kit (Roche) as described [26], using primers A and B for syncytin and primers for HER-2 [26]. Syncytin mRNA levels were expressed relative to HER-2 mRNA levels. 


\section{Results}

\section{Syncytin is expressed by breast cancer cells and ASCT-2 is expressed both by cancer cells and by endothelial cells}

Reverse transcriptase-polymerase chain reaction (RT-PCR) of RNA isolated from MCF-7 and MDA-MB-231 breast cancer cells revealed amplicons of the expected size (400 base pairs) from both cancer cell lines (Fig. 1A). No amplicon was obtained from endothelial cells. Exclusion of reverse transcriptase and use of intron-spanning primers documented lack of genomic contamination. Sequencing of the RT-PCR products revealed $99 \%$ (MCF-7) and 82\% (MDA-MB-231) homology to syncytin. Immunoblotting using an antiserum against the extracellular domain of syncytin revealed a 62-kDa immunoreactive band in extracts of MCF-7 and MDA-MB-231 cells (Fig. 1B), corresponding in size to a syncytin component present in the placenta [27].

Immunofluorescence staining of non-permeabilized MCF-7 cells showed that syncytin immunoreactivity was exposed on the external cell surface (Fig. 1C). Immunocytochemistry showed that 63 out of 165 human breast cancer specimens (38\%) contained cancer cells that reacted for syncytin (Fig. 2A). A variable number of cancer cells were stained in individual tumors. Preabsorption of the antibody with the immunizing peptide eliminated staining (Fig. 2B).

Immunofluorescence staining demonstrated expression of the syncytin receptor ASCT-2 in endothelial cells as well as in cancer cells (Fig 2C). Absorption of the antiserum with the immunizing peptide eliminated staining. Double immunofluorescence for ASCT-2 and CD31 confirmed that endothelial cells expressed ASCT-2. RT-PCR of RNA extracted from human endothelial (HUVECs) and breast cancer (MCF-7, MDA-MB231) cells revealed an amplicon of the size expected from human ASCT-2 mRNA (445 
base pairs; data not shown). Additionally, RT-PCR of RNA from bovine endothelial (CPAE) cells demonstrated an amplicon of the size expected (491 base pairs) from bovine ASCT-2 mRNA (Fig 1A).

\section{Antisense downmodulation of syncytin expression inhibits fusions between breast cancer cells and endothelial cells}

Treatment of MCF-7 cells with a phosphorthioate-protected syncytin antisense oligonucleotide decreased syncytin mRNA levels (by $33.8 \pm 5.0 \%$ as compared to a control, scrambled phosphorthioate oligo) and protein levels (by $38.2 \pm 13.2 \%$ ). Levels were expressed relative to control mRNA/protein (HER-2) and therefore reflect a specific effect on syncytin expression. Transfected cells were co-cultured with HUVECs or with CPAE cells for $24 \mathrm{~h}$ and were then assayed for the frequency of cancer-endothelial cell fusion [5]. Briefly, co-cultures were fixed and triple-stained for vimentin (which is always expressed by HUVECs and by CPAE cells, but never by MCF-7 cells), cytokeratin (which is always expressed by MCF-7 cells, but never by HUVECs or CPAE cells) and DNA. After $24 \mathrm{~h}$ of co-culture, fused cells expressing both vimentin and cytokeratin and containing two or more nuclei were regularly observed in control co-cultures (Fig. 3A). Such cells were never observed when MCF-7, HUVECs or CPAE cells were cultured alone. Quantitations of the frequency of fused cells in blind-coded specimens documented that antisense treatment significantly ( $<<0.001)$ inhibited cancer-endothelial cell fusions (Fig. 3B).

\section{An inhibitory syncytin peptide inhibits fusions between breast cancer cells and endothelial cells}

The previous results indicated that downmodulation of syncytin expression by an antisense oligonucleotide, which previously has been evaluated in placental cells [25], 
inhibited fusions between MCF-7 cells. However, since it is not always possible to conclude from antisense experiments that the effect observed reflects a direct action of the molecule targeted, we also examined effects of a syncytin peptide sequence, which previously has been shown to inhibit syncytin-mediated fusions [24]. MCF-7 cells, cocultured with HUVECs in the presence of this peptide, showed a highly significant $(p<0.001)$ reduction in the number of cell fusions as compared to control cells that were exposed to equivalent concentrations of a control peptide (C-peptide) or were left untreated (Fig. 3). There was no difference in the number of fusions between cells grown in the presence or absence of $\mathrm{C}$-peptide $(\mathrm{p}=0.6)$. Together, these results provide strong evidence that syncytin is directly involved in breast cancer-endothelial cell fusions. 


\section{Discussion}

These data are the first to show that syncytin is expressed by human cancer cells. Thus, two human breast cancer cell lines and over a third of human ductal breast cancers were found to express syncytin. Previously, studies have demonstrated that syncytin is expressed in the placenta, testicles [2, 3] and brain [28]. In order for syncytin to induce cell fusions, fusion partners must express an appropriate receptor such as the D-type retroviral receptor, ASCT-2 [2]. We therefore examined breast cancers and cultured endothelial cells for expression of ASCT-2 and detected expression in both cell types in vitro and in vivo. These results show that the molecular requirements for syncytinASCT-2-mediated fusions are present in a significant number of breast cancer patients. Previously, we have documented that human breast cancer cells fuse with endothelial cells in a system validated by chromosomal and protein markers [5]. We therefore used this system to test whether syncytin was involved in mediating cancer-endothelial cell fusions. Syncytin expression was downmodulated by an antisense oligonucleotide sequence, which previously has been shown to specifically decrease syncytin expression and to inhibit fusions between placental trophoblasts [26]. The specificity of downmodulation was confirmed by simultaneous measurement of mRNA and protein levels of a reference (HER-2). Assays for cell fusions revealed that syncytin antisense treatment significantly inhibited cancer-endothelial cell fusions. Additionally, we also found that a syncytin inhibitory peptide [24] inhibited cancer-endothelial cell fusions. These results form definite evidence that syncytin is involved in mediating fusions between breast cancer cells and endothelial cells and suggest that spontaneous, syncytin-mediated fusions may occur in human breast cancer.

It may be speculated that cancer-endothelial cell fusions occur in vivo when syncytinproducing cancer cells come into direct contact with ASCT-2 expressing endothelial cells, e. g. during extravasation. Depending upon the genetic make-up of the fusion 
partners such fusions may either result in the activation of tumor suppressors contributed by the normal fusion partner [14-18] or may result in hybrids with more aggressive properties [4, 20-22]. 


\section{References}

1. Witze E, Rothman JH. Cell fusion: An EFFicient sculptor. Curr. Biol. 2002;12:R467-69.

2. Blond J. L., Lavillette D., Cheynet V., Bouton O., Oriol G., Chapel-Fernandes S. et al. (2000) An envelope glycoprotein of the human endogenous retrovirus HERV-W is expressed in the human placenta and fuses cells expressing the type D mammalian retrovirus receptor. J. Virol. 74: 3321-3329

3. Mi S., Lee X., Li X., Veldman G. M., Finnerty H., Racie L. et al. (2000) Syncytin is a captive retroviral envelope protein involved in human placental morphogenesis. Nature 403: 785-789

4. Duelli D. and Lazebnik Y. (2003) Cell fusion: a hidden enemy? Cancer Cell 3: 445448

5. Mortensen K., Lichtenberg J., Thomsen P. D. and Larsson, L.-I. (2004) Spontaneous fusion between cancer cells and endothelial cells. Cell. Mol. Life Sci. 61: 2125-2131

6. Barski G. and Cornefert F. (1962) Characteristics of "hybrid"-type clonal cell lines obtained from mixed cultures in vitro. J. Natl. Cancer Inst. 28: 801-821

7. Busund L. T. R., Killie M. K., Bartnes K., Olsen R. and Seljelid R. (2002) Spontaneous hybridization of macrophages and Meth A sarcoma cells. Int. J. Cancer 98: 573-581

8. Miller F. R., McInerney D., Rogers C. and Miller B. E. (1988) Spontaneous fusion between metastatic mammary tumor subpopulations. J. Cell. Biochem. 36: 129-136

9. Wakeling W. F., Greetham J. and Bennett D. C. (1994) Efficient spontaneous fusion between some co-cultured cells, especially murine melanoma cells. Cell Biol. Int. 18: $207-210$ 
10. Fortuna M. B., Dewey M. J. and Furmanski P. (1990) Enhanced lung colonization and tumorigenicity of fused cells isolated from primary MCA tumors. Cancer Lett. 55: $109-114$

11. Goldenberg D. M., Pavia R. A. and Tsao M.C. (1974) In vivo hybridisation of human tumor and normal hamster cells. Nature 250: 649-651

12. Larizza L., Schirrmacher V., Graf L., Pfluger E., Peres-Martinez M. and Stohr M. (1984) Suggestive evidence that the highly metastatic variant ESb of the T-cell lymphoma Eb is derived from spontaneous fusion with a host macrophage. Int. J. Cancer 34: 699-702

13. Munzarova M., Lauerova L. and Capkova J. (1992) Are advanced malignant melanoma cells hybrids between melanocytes and macrophages? Melanoma Res. 2: $127-129$

14. Harris H., Miller O. J., Klein G., Worst P. and Tachibana T. (1969) Suppression of malignancy by cell fusion. Nature 223: 363-368

15. Harris H. (1988) The analysis of malignancy by cell fusion: the position in 1988. Cancer Res. 48: 3302-3306

16. Stanbridge E. J. (1976) Suppression of malignancy in human cells. Nature 260: 1720

17. Wiener F., Klein G. and Harris H. (1974) The analysis of malignancy by cell fusion. V. Further evidence of the ability of normal diploid cells to suppress malignancy. J. Cell Sci. 15: 177-183

18. Anderson M. J. and Stanbridge E.J. (1993) Tumor suppressor genes studied by cell hybridization and chromosome transfer. FASEB J 7: 826-833 
19. Kohler G. and Milstein C. (1975) Continuous cultures of fused cells secreting antibody of predefined specificity. Nature 256: 495-497

20. Friedl P. (2005) New mechanisms of plasticity in cancer? Lancet Oncology 6: 916918

21. Pawelek J. M. (2005) Tumor-cell fusion as a source of myeloid traits in cancer. Lancet Oncology 6: 988-993

22. Duelli D. M., Hearn S., Myers M. P., Lazebnik Y. (2005) A primate virus generates transformed human cells by fusion. J. Cell Biol. 171: 493-503

23. Mortensen K., Skouv J., Hougaard D. M. and Larsson L.-I. (1999) Endogenous endothelial cell nitric oxide synthase modulates apoptosis in cultured breast cancer cells and is transcriptionally regulated by p53. J. Biol. Chem. 274: 37679-37684

24. Chang C, Chen PT, Chang GD, Huang CJ, Chen H (2004) Functional characterization of the placental fusogenic membrane protein syncytin. Biol. Reprod. 71: 1956-1962

25. Frendo J. L., Olivier D., Cheynet V., Blond J. L., Bouton O., Vidaud M. et al. (2003) Direct involvement of HERV-W Env glycoprotein in human trophoblast cell fusion and differentiation. Mol. Cell. Biol. 23: 3566-3574

26. Larsson L.-I. (2004) Novel actions of tyrphostin AG 879: Inhibition of RAF-1 and HER-2 expression combined with strong antitumor effects on breast cancer cells. Cell. Mol. Life Sci. 61: 2624-2631

27. Smallwood A., Papageorghiou A., Nicolaides K., Alley M. K., Jim A., Nargund G. et al. (2003) Temporal regulation of the expression of syncytin (HERV-W), maternally imprinted PEG10 and SGCE in human placenta. Biol. Reprod. 69: 286293 
28. Antony J. M., van Marle G., Opii W., Butterfield D. A., Mallet F., Yong V. W. et al. (2004) Human endogenous retrovirus glycoprotein-mediated induction of redox reactants causes oligodendrocyte death and demyelination. Nature Neurosci. 7: 1088-1095 


\section{Acknowledgements}

Grant support was from the Danish Cancer Society, the Danish Medical Research

Council and the Lundbeck Foundation (to LIL) and Aase and Ejnar Danielsens Fond (to IJC). We thank P. D. Thomsen for use of the LightCycler. 


\section{LEGENDS TO FIGURES}

Fig. 1. Expression of syncytin by breast cancer cells and of ASCT-2 by endothelial cells. a. RT-PCR detects an amplicon of the size expected for syncytin (400 bp) in extracts of MCF-7 (lane 1) and MDA-231 cells (lane 2). Exclusion of RT yields no amplicon (shown for MCF-7 cells; lane 3) and ASCT-2 primers produce an amplicon of 491 base pairs from CPAE cell extracts (lane 4). Positions of a base ladder (lane 5) are shown to the right. b. Immunoblotting detects a 62-kDa band in extracts of MCF-7 cells. c. Immunofluorescence staining for syncytin of live (antibody-impermeable) MCF-7 cells. Note surface expression of syncytin.

Fig 2. Immunocytochemical demonstration of syncytin and syncytin receptor (ASCT-2) expression in breast cancer. a. Immunoperoxidase staining for syncytin in human breast cancer specimen. Nuclei are stained blue with haematoxylin. Note syncytin immunoreactivity (brown) in scattered tumor cells. b. Adjacent section to the one shown in a, stained with antigen-preabsorbed antiserum. Note absence of staining. c. Human breast cancer specimen triple-stained for ASCT-2 (green fluorescence), the endothelial marker CD31 (red fluorescence) and DNA (blue fluorescence). Arrows point to endothelial cells that stain both for ASCT-2 and CD31 and, thus, emit orange fluorescence. To the right in the image, tumor cells that react for ASCT-2, but not for CD31, emit green fluorescence.

Fig. 3. Fusions between breast cancer cells and endothelial cells depend upon syncytin.

a. Control co-culture of MCF-7 and endothelial cells, triple-stained for vimentin (green), cytokeratin (red) and DNA (blue). Note a hybrid cell expressing both vimentin and cytokeratin (yellow). b. Quantitation of fused cells in co-cultures of MCF-7 and CPAE cells or of MCF-7 cells and HUVECs shows that the frequency of cancer-endothelial cell fusions is inhibited by syncytin antisense, but not by control (scrambled) oligonucleotide treatment. c. Treatment with an inhibitory syncytin peptide inhibits 
fusions between MCF-7 cells and HUVECs while a control peptide (C-peptide) has no effect. Three stars denote $\mathrm{p}<0.001$. 


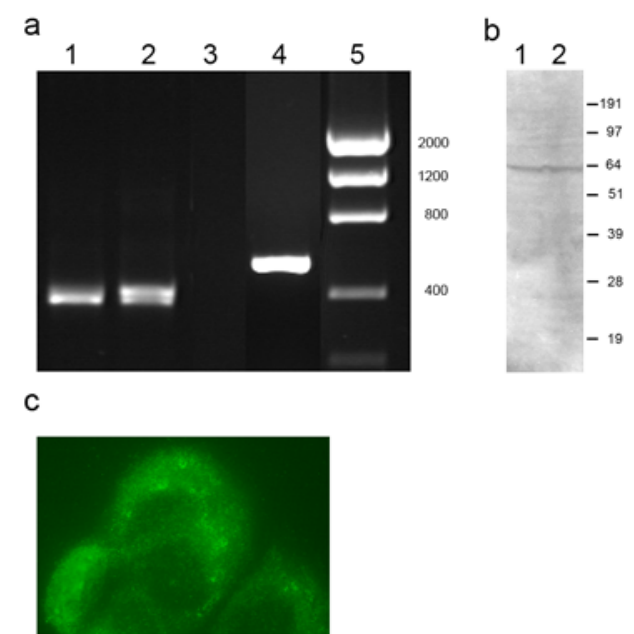

Fig. 1 

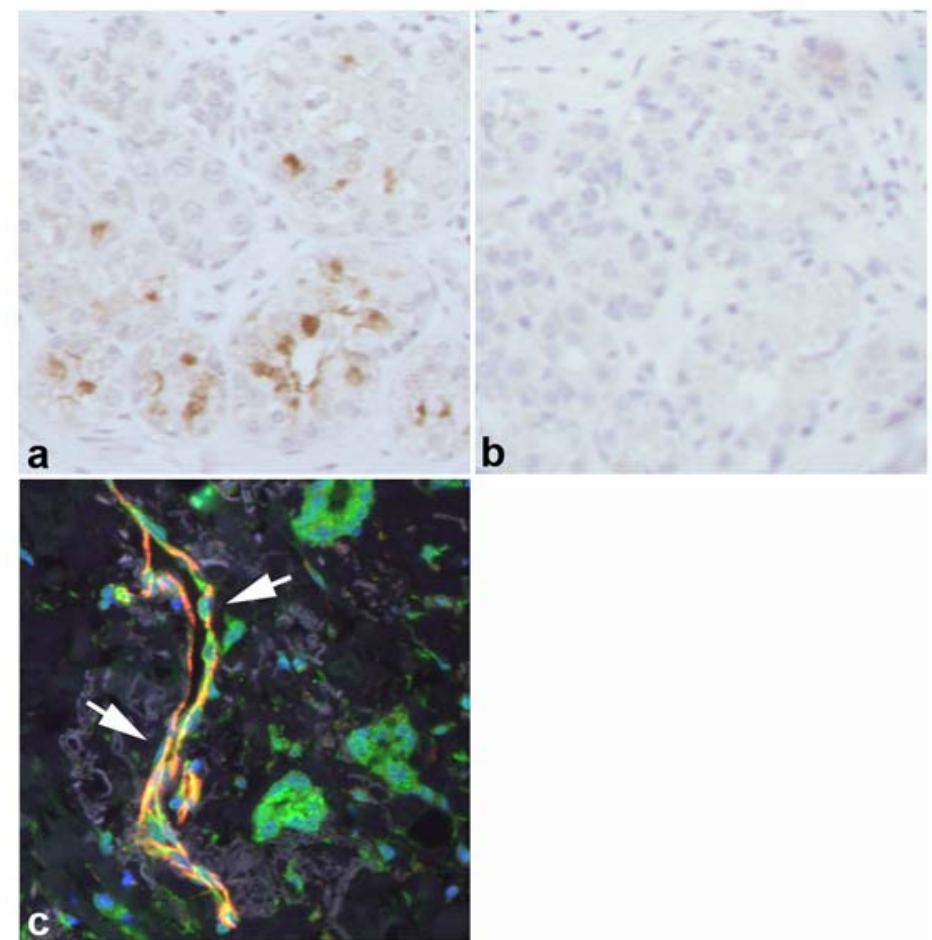

Fig. 2 


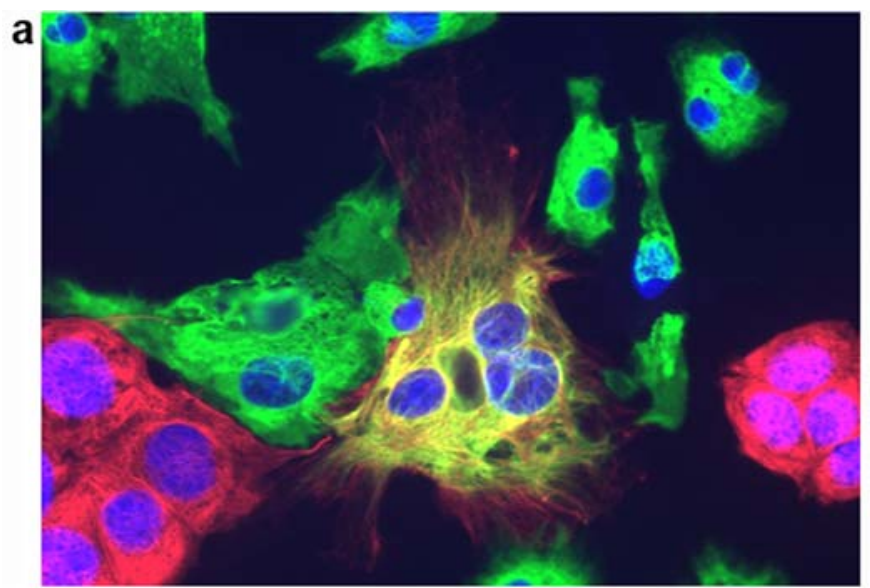

b

Antisense downmodulation

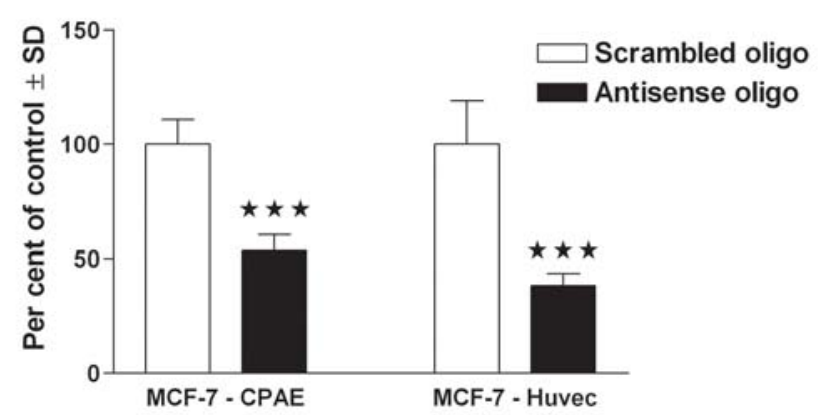

C

Peptide inhibition

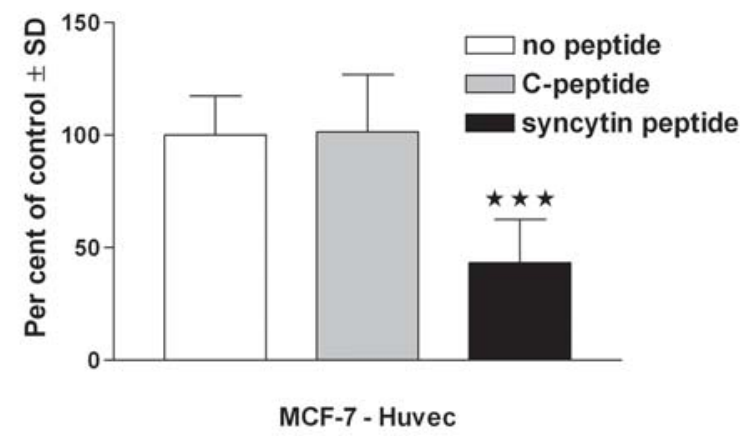

Fig. 3 